\title{
HIGHLIGHTS
}

PROSTATE CANCER

\section{Is fracture prevention therapy cost-effective in patients with prostate cancer treated with ADT?}

Androgen deprivation therapy (ADT) is widely used as an adjuvant treatment in patients with locally advanced or high-risk localized prostate cancer. As androgen deficiency is associated with decreased bone mineral density (BMD), and as prostate cancer occurs most frequently in people of advanced age, men receiving ADT are particularly susceptible to development of osteoporosis and fractures. Some authors have recommended BMD assessment followed by antiresorptive therapy only for patients with high fracture risk; others have proposed routine administration of antiresorptive agents to all patients receiving ADT, regardless of BMD. Researchers at the Memorial SloanKettering Cancer Center investigated the cost-effectiveness of these strategies using a computer simulation approach.

The team, led by Kouta Ito, developed a Markov state-transition model that simulated prostate cancer progression and hip fracture incidence. The hypothetical cohort included men aged 70 years with T2c to T4N0 prostate cancer undergoing 2 years of ADT after radiation therapy. Dual-energy X-ray absorptiometry was the assumed method of BMD measurement, and alendronate was the sole antiresorptive agent used in the model. The three strategies tested were as follows: no BMD test and no alendronate treatment (the reference strategy); a single BMD test followed by selective alendronate treatment only in patients with osteoporosis; and universal alendronate treatment with no BMD test.

The analysis indicated that selective alendronate treatment following BMD measurement was the only cost-effective strategy in the base-case cohort. Universal alendronate treatment, although above acceptable cost thresholds, became reasonably cost-effective when the basecase population was adjusted to be older,

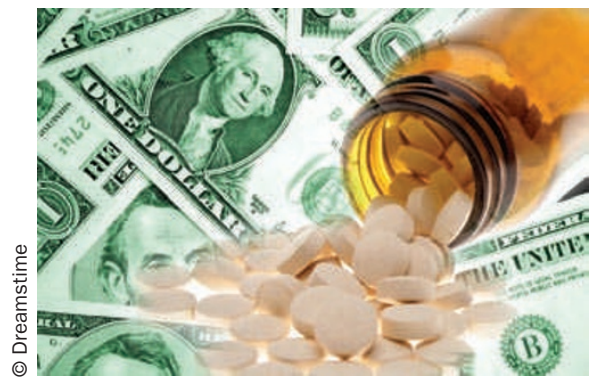

have a history of fractures, lower BMD, or if the price of alendronate was lower.

A limitation of the study was that there is currently no hard evidence that bisphosphonates decrease the risk of fractures in patients reciving ADT for prostate cancer.

\section{Nick Warde}

Original article Ito, K. et al. Cost-effectiveness of fracture prevention in men who receive androgen deprivation therapy for localized prostate cancer. Ann. Intern. Med. 152, 621-629 (2010) 